\title{
Results of isolated posterolateral corner reconstruction
}

\author{
Lawrence Camarda $\cdot$ Vincenzo Condello • \\ Vincenzo Madonna $\cdot$ Fabrizio Cortese • \\ Michele D'Arienzo • Claudio Zorzi
}

Received: 16 October 2009/ Accepted: 8 February 2010/Published online: 13 March 2010

(C) Springer-Verlag 2010

\begin{abstract}
Background Isolated posterolateral corner (PLC) tears are relatively rare events. Various surgical techniques to treat posterolateral knee instability have been described; because surgical results are linked to cruciate reconstructions it has been difficult to date to define whether one surgical procedure has better prognosis than another. The goal of this study is to determine the clinical outcome of PLC reconstruction following fibular-based technique.

Materials and methods We retrospectively evaluated a case series of patients who received isolated PLC reconstruction between March 2005 and January 2007. Ten patients were surgically treated for isolated injuries and were available for follow-up; average patient age was 27.4 years (range 16-47 years). All patients were treated following the fibular-based technique: double femoral tunnel was performed in six patients, while in the remaining four patients, the reconstruction of the PLC was performed with a single femoral tunnel. Six patients had semitendinosus allograft and four had semitendinosus autograft. All patients had the same evaluation and the same rehabilitation protocol.

Results Mean follow-up was 27.5 months (range $18-40$ months). Mean range of motion (ROM) was $143.5^{\circ}$ for flexion (range $135-150^{\circ}$ ) and $0.5^{\circ}$ for extension (range
\end{abstract}

L. Camarda $(\square) \cdot$ M. D’Arienzo

Clinica Ortopedica e Traumatologica,

Policlinico Universitario "P.Giaccone",

Via del Vespro 125, 90127 Palermo, Italy

e-mail: lawrencecamarda@unipa.it

V. Condello · V. Madonna $\cdot$ F. Cortese $\cdot$ C. Zorzi

Ospedale Sacro Cuore Don Calabria,

Centro di Chirurgia del Ginocchio,

Via Settembrini, Negrar, Verona, Italy $\left.0-3^{\circ}\right)$. Three patients showed $1+$ on varus stress test, while on Dial test another three patients showed $10^{\circ}$ reduction of external rotation compared with contralateral knee. The average Lysholm score was 94 points (range 83-100), and the mean International Knee Documentation Committee (IKDC) subjective result was 88.48 (range 74-96.5). Based on Lysholm score, the results were excellent in eight knees and good in two knees. On IKDC evaluation, two patients were grade $\mathrm{A}$ and eight were grade $\mathrm{B}$. No significant difference in clinical results was observed between single and double femoral tunnel.

Conclusion Fibular-based technique showed good results in terms of clinical outcome, restoring varus and rotation stability of knees in treatment of chronic isolated PLC injury.

Keywords Posterolateral corner (PLC) reconstruction . Fibular collateral ligament - Knee ligament reconstruction . Popliteofibular ligament

\section{Introduction}

In the past the posterolateral corner (PLC) of the knee was described as the "the dark side of the knee" [1]. The definition is well suited to the posterolateral structures of the knee. For too long neglected, this anatomical region has recently attracted the attention of researchers and scholars, for a long time focused on study of the anterior cruciate ligament (ACL). However, analysis of the results and failures of ACL reconstructive surgery have permitted greater understanding of the complex biomechanics of the knee and injuries associated with cruciate tears.

Over the past 15 years, numerous cadaveric studies have focused on anatomy and biomechanics of the PLC of the 
knee, enabling greater knowledge of this anatomical region. Following these studies, it has been clarified that the fibular collateral ligament (FCL), the popliteofibular ligament (PFL), and the popliteal tendon (PT) play a main role in resisting external rotation, varus rotation, and posterior tibial translation $[2,3]$. Specifically, the FCL and PFL represent the main static stabilizers of the PLC, working as major restraints to primary varus and external rotation at lower angles of knee flexion $\left(30^{\circ}\right)$, whereas the popliteus muscle-tendon unit functions as the dynamic stabilizer of external rotation and posterior tibial translation at greater knee flexion angles [3-5].

Many authors recommend operative reconstruction in symptomatic chronic PLC tears not responding to conservative treatment. However, because of different surgical techniques, controversies still exist regarding which surgical procedure would give the best results. Moreover, sparse literature data are available concerning in vivo results of this surgical procedure, and all of them describe PLC surgery performed in settings with associated cruciate ligament injury [6].

The purpose of this study is to evaluate the clinical outcome of patients who underwent isolated posterolateral corner reconstruction performed according to the fibularbased technique. Our hypothesis is that the fibular-based technique could restore varus and rotation stability in knees with chronic isolated PLC injury.

\section{Materials and methods}

Between March 2005 and January 2007, 12 consecutive patients were surgically treated for chronic isolated posterolateral corner injury. Two patients were lost to followup because they were living abroad. Ten patients were available for follow-up (nine men and one woman). Eight patients underwent surgical reconstruction between 4 and 12 months after injury, and two patients underwent surgery more than 12 months after injury. Before surgery all patients complained of knee pain on the lateral side and instability even after a rehabilitation program was performed. In four patients the diagnosis of PLC injury was made at 3 months from the injury. The mechanisms of injury included five motorcycle crashes and five sportrelated injuries.

In addition to cruciate ligament tests, a specific posterolateral corner evaluation was performed. The Dial test (at $30^{\circ}$ and $90^{\circ}$ knee flexion) and the varus stress test (at $0^{\circ}$ and $30^{\circ}$ knee flexion) were performed, respectively, in prone and supine position. The results of these tests were compared with those of the contralateral, uninvolved knee and were considered normal when no difference was found between the involved and uninvolved knee. Instability was graded from 0 to $3+$ (Table 2) [7]. The Dial test and the varus stress test were performed also preoperatively with the patient under spinal or general anesthesia. Furthermore, arthroscopic evaluation was performed to detect more than $1 \mathrm{~cm}$ of lateral compartment opening with application of varus knee stress ("drive-through" sign) [8]. Surgical reconstruction was performed upon positivity of the Dial test, varus stress test, magnetic resonance imaging (MRI) examination, and if the drive-through sign was present.

Before surgical reconstruction all patients underwent MRI and long leg weight-bearing X-ray examination to exclude cruciate ligament tear, previous fracture, and limb malalignment. However, in three patients we found a posterior cruciate ligament (PCL) partial lesion. In these patients, radiofrequency ligament shrinkage was performed rather than surgical reconstruction. This choice was made on the basis of the clinical knee examination (tibiofemoral step-off $<5 \mathrm{~mm}$ on posterior drawer test), the MRI, and the arthroscopic assessment ligament view. Other associated injuries included one medial meniscal tear treated through partial meniscectomy, while another two patients presented grade II Outerbridge patellar chondropathy and were not treated (Table 1); at time of injury, no patients had any associated fractures. The exclusion criteria of the study were previous knee surgery and associated cruciate ligament injury that both clinically and arthroscopically required surgical reconstruction.

All patients were treated by fibular-based technique reconstruction. Semitendinosus tendon was used in all patients, with the source being autogenous in four and allograft in six, due to allograft availability. All reconstruction procedures were performed by the senior surgeon. Following McGuire's technique [9], a single femoral tunnel was performed on four patients, while in the remaining six patients, the reconstruction of the PLC was performed with a double femoral tunnel, as described by Arciero [10] (Figs. 1, 2).

The patient was placed supine on the operating table. Tourniquet was placed high up on the thigh, and an arthroscopic assessment view was performed. PLC reconstruction was done using semitendinosus tendon. The tendon graft was tubularized using a no. 2 nonabsorbable suture (Fiberwire; Arthrex, Naples, FL) and was sized to pass through a $6-\mathrm{mm}$ tunnel, measuring $20-24 \mathrm{~cm}$ in length.

With the knee flexed at $90^{\circ}$, the initial lateral exposure was made through a skin incision over the lateral aspect of the knee. A straight-line incision was performed just from the posterior aspect of the lateral epicondyle to just the distal part of the fibular head. A 4-cm horizontal fascial incision was performed posterior to the fibular head, just anterior to and in line with the biceps tendon. Thereafter, the fibers of the lateral gastrocnemius muscle were elevated 
Table 1 Results of isolated posterolateral corner reconstruction

\begin{tabular}{|c|c|c|c|c|c|c|c|c|c|c|}
\hline Patient & $\begin{array}{l}\text { Age } \\
\text { (years) }\end{array}$ & $\begin{array}{l}\text { Follow-up } \\
\text { (months) }\end{array}$ & Associate tears & Graft & Tunnel & VAS & Lysholm & IKDC & $\begin{array}{l}\text { Varus } \\
\text { stress }\left(30^{\circ}\right)\end{array}$ & $\begin{array}{l}\text { Dial } \\
\text { test } 30^{\circ}\end{array}$ \\
\hline 1 & 30 & 33 & - & Allograft & Double & 1 & 92 & $85-(B)$ & 0 & $0\left(<10^{\circ}\right)$ \\
\hline 2 & 36 & 18 & Patellar chondropathy & Allograft & Single & 1 & 96 & 71.3-(B) & 1 & 0 \\
\hline 3 & 47 & 29 & - & Allograft & Double & 2 & 86 & 83.9-(B) & 0 & 0 \\
\hline 4 & 25 & 18 & PCL & Autologous & Double & 1 & 100 & 95.4-(B) & 0 & 0 \\
\hline 5 & 16 & 33 & - & Autologous & Double & 0 & 100 & 97.7-(A) & 1 & 0 \\
\hline 6 & 16 & 35 & PCL & Allograft & Single & 0 & 99 & 96.5-(A) & 0 & 0 \\
\hline 7 & 19 & 27 & LM & Allograft & Double & 3 & 83 & 74.7-(B) & 1 & 0 \\
\hline 8 & 17 & 21 & Patellar chondropathy & Autologous & Double & 2 & 90 & 96.5-(B) & 0 & $0\left(<10^{\circ}\right)$ \\
\hline 9 & 47 & 40 & - & Allograft & Single & 0 & 95 & 89.6-(B) & 0 & 0 \\
\hline 10 & 21 & 21 & PCL & Autologous & Single & 0.5 & 99 & 94.2-(B) & 0 & $0\left(<10^{\circ}\right)$ \\
\hline
\end{tabular}

Three patients presented $10^{\circ}$ reduction of tibial external rotation compared with contralateral knee

$P C L$ posterior cruciate ligament, $L M$ lateral meniscus

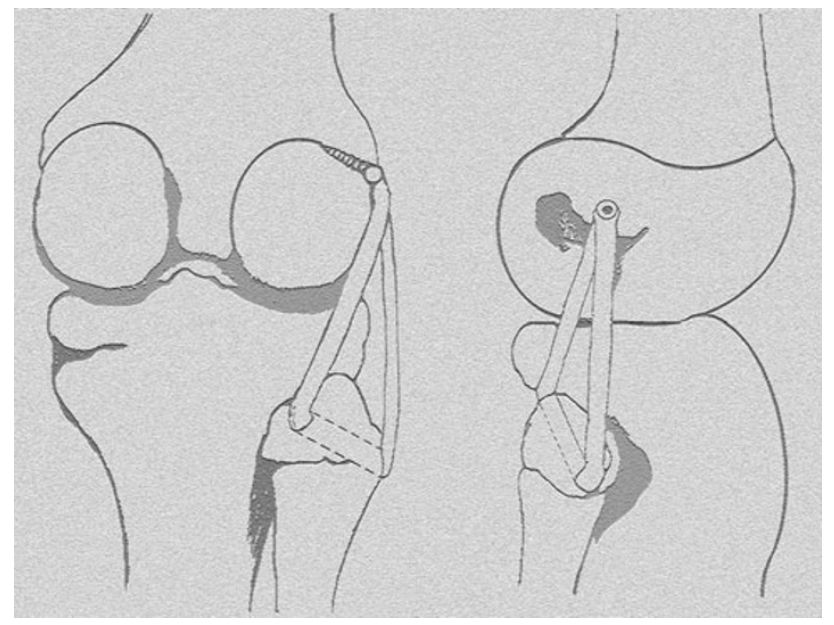

Fig. 1 Single femoral tunnel technique, as described by McGuire

from the fibula, exposing the posterior aspect of the fibular head. Furthermore, a small vertical incision was made over the anterolateral aspect of the proximal fibula to expose the origin of the fibular collateral ligament.

Protecting the peroneal nerve posteriorly with a teaspoon, a guidewire was then passed from just anteriorly and distally to the insertion of the FCL and directed proximally and medially to exit the posterior aspect of the fibula adjacent to the proximal tibiofibular joint (Fig. 3). Then, a 6-mm tunnel was drilled and the graft was then passed through the tunnel. At this point, the lateral femoral epicondyle was identified and a 4-cm iliotibial band incision was performed over this point. The FCL attachment point was anatomically located, and a guidewire was introduced just anteriorly to the central origin of the FCL. The guidewire was slightly inclined from posterior to anterior and was directed proximomedially to the medial epicondyle and adductor tubercle. The guidewire was

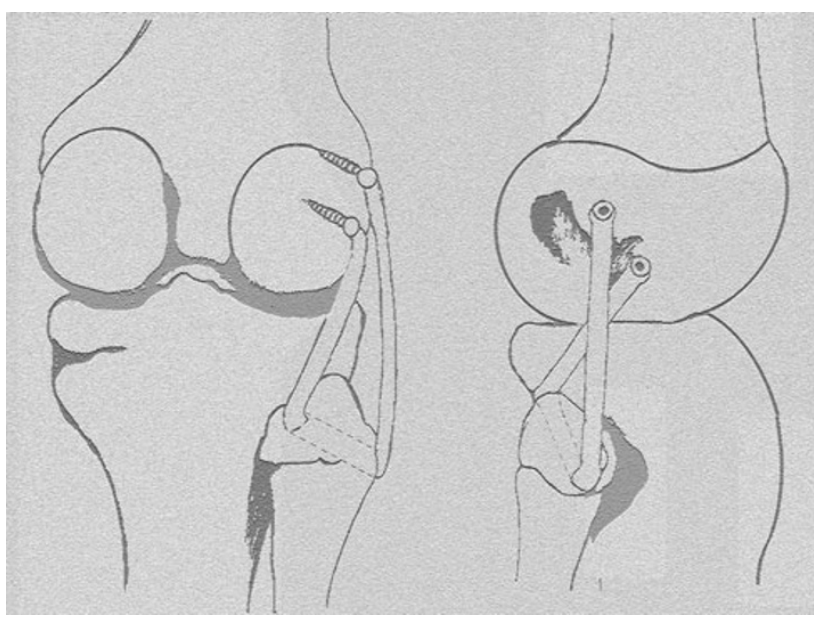

Fig. 2 Double femoral tunnel following Arciero's technique

brought out percutaneously to the medial side of the knee, and then an 8-mm tunnel was reamed to a depth of $35 \mathrm{~mm}$ (FCL tunnel). At this point, both ends of the graft were passed under the iliotibial band and then through the femoral tunnel. The graft was tensioned with the knee flexed at $30^{\circ}$, internally rotated and slightly valgus. Graft fixation on the femoral tunnel was performed with a 9-mm bioabsorbable screw (BioRCI-HA; Smith \& Nephew, Andover, MA).

Following the double femoral socket technique described by Arciero [10], in six patients one more femoral tunnel (PFL tunnel) was reamed, $18 \mathrm{~mm}$ distally and anteriorly from the FCL tunnel to a depth of $35 \mathrm{~mm}$ (Fig. 4). Both tunnels were sized $6 \mathrm{~mm}$. The anterior limb of the graft was passed deep to the superficial layer of the iliotibial band and then through the FCL tunnel. Instead, the posterior limb of the graft was passed through the popliteal hiatus and then transferred into the PFL tunnel. 


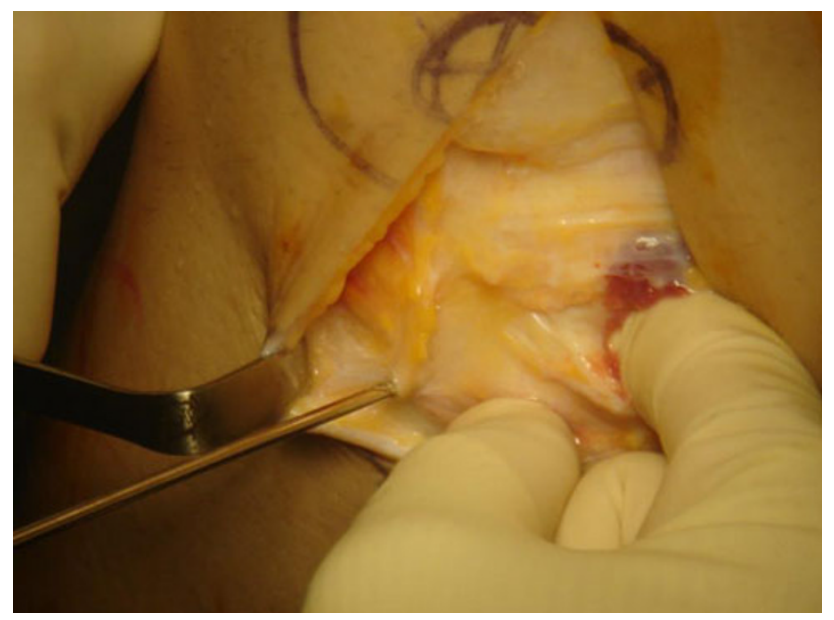

Fig. 3 The guidewire is passed from anterior-distally to the insertion of the FCL and directed posterior-medially

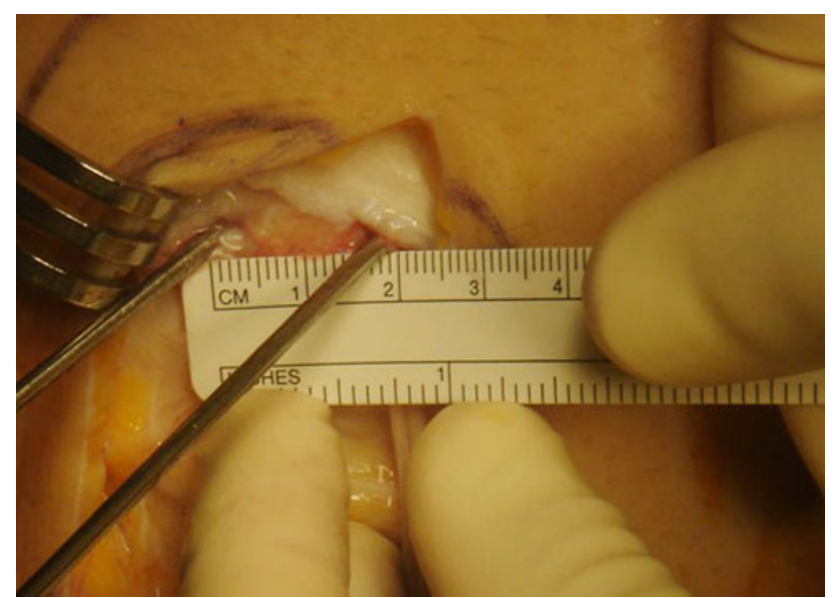

Fig. 4 The popliteal femoral guidewire and the fibular femoral guidewire are parallel. The separation should be $18 \mathrm{~mm}$ for correct anatomical reconstruction

The graft was tensioned with the knee flexed at $30^{\circ}$, internally rotated and slightly valgus. The graft fixation on the femoral tunnels was performed with two 7-mm bioabsorbable screws.

\section{Rehabilitation}

All patients underwent the same rehabilitation program. The operated knee was immobilized in a postoperative hinged knee brace locked at $0^{\circ}$ for 3 weeks. Passive ROM began at the third week postoperatively, and at the fourth week the brace was unlocked to achieve a range of motion of $0-110^{\circ}$. In this phase the patient started partial weight bearing, as tolerated with crutches. Full weight bearing was allowed from the seventh week postoperatively. The high knee brace was kept until the 12th week to protect the knee from varus stress and from knee hyperextension, and to permit graft integration. During the whole rehabilitation period patients were instructed to limit excessive foot and tibial rotation.

Patients returned to regular daily activities at the fourth month, but running was not permitted until 5 months after surgery. Contact sports were permitted after 7 months postoperatively.

Follow-up evaluation

All examinations and results were evaluated by a single independent examiner not involved in surgical treatment. Knee function was evaluated according to Lysholm/Tegner score and the International Knee Documentation Committee (IKDC) subjective and objective forms. In addition, a visual analog scale (VAS) was submitted to evaluate residual knee pain affecting the patient during the last 2 weeks.

On clinical examination, the results obtained in the injured knee were compared with those of the uninjured side. Extension of the knee was measured with the patient in supine position, while flexion was measured in prone position. During clinical examination of the knee, we paid particular attention to assessing the stability of the posterolateral corner complex. The varus stress test was performed in supine position at $0^{\circ}$ and $30^{\circ}$, while the Dial test of the treated knee was performed in prone position at knee flexion of $30^{\circ}$ and $90^{\circ}$. The measurement was obtained by a goniometer, and the results were recorded and graded on a 0 to $3+$ scale [11] (Table 2). Furthermore, we asked each patient to classify his/her knee condition as follows: greatly improved, improved, no change, slightly worse, or greatly worse.

All subjects gave informed consent prior to inclusion to the study, which was approved by the local ethical committee. The study was performed in accordance with the Declaration of Helsinki as revised in 2000.

\section{Statistical analysis}

Statistical analysis was conducted by an independent statistician who was not associated with the surgical team. Clinical results of Dial test and varus stress test were compared with the Lysholm score and IKDC score using

Table 2 Classification of posterolateral instability

\begin{tabular}{llll}
\hline & Varus stress test $0^{\circ}-30^{\circ}$ & Dial test $30^{\circ}$ & Result \\
\hline 0 & No laxity & $<5^{\circ}$ & Successful result \\
$1+$ & $5^{\circ}$ of laxity & $>5^{\circ} ;<10^{\circ}$ & Successful result \\
$2+\quad 10^{\circ}$ of laxity & $>10^{\circ} ;<15^{\circ}$ & Failure result \\
$3+\quad 15^{\circ}$ or more of laxity & $>15^{\circ}$ & Failure result \\
\hline
\end{tabular}


paired simple regression analysis. For all analyses a $P$ value of $\leq 0.05$ was considered significant. Data were analyzed using SPSS statistical software release 13.0 (SPSS Inc., Chicago, IL) for Windows.

\section{Results}

Average patient age at surgery time was 27.4 years (range 16-47 years). There were nine men and one woman. The average follow-up period was 27.5 months (range 1840 months). In six patients submitted to PLC reconstruction a semitendinosus allograft was used, while a semitendinosus autologous was used in four patients. In six patients a double femoral tunnel was performed, while in the remaining four patients the PLC reconstruction was performed following the single femoral tunnel technique (Table 1).

No patients had significant flexion contracture. The average $\mathrm{ROM}$ was $143.5^{\circ}$ for flexion (range $135-150^{\circ}$ ) and $0.5^{\circ}$ for extension (range $0-3^{\circ}$ ). However, on varus stress test $\left(0-30^{\circ}\right)$ three patients showed $1+$ while the other seven showed normal lateral opening (0) compared with the contralateral knee (average 0.3). The Dial test performed at $30^{\circ}$ showed that, following the 0 to $3+$ system, all treated knees were classified as normal (0). However, three patients presented $10^{\circ}$ reduction of external rotation compared with the contralateral knee. No differences between the two knees were observed by Dial test performed at $90^{\circ}$ knee flexion. The average Lysholm knee score was 94 points (range 83-100). Based on the Lysholm knee scoring system, the results were excellent in eight knees and good in two knees. The objective results of the IKDC score were normal (A) in two patients and nearly normal (B) in eight patients. No abnormal or severely abnormal results $(\mathrm{C}, \mathrm{D})$ were observed in this study. The mean postoperative IKDC subjective score was 88.48 (range 74-97.7).

No correlation was found on comparing the clinical results on Dial test with the Lysholm score $(P=0.917)$ or IKDC score $(P=0.489)$. Similarly, no correlation was found comparing the clinical results on varus stress test with Lysholm score $(P=0.180)$ or IKDC score $(P=0.118)$.

Postoperative complications were observed in two patients. A 47-year-old man [follow-up (FU) 32 months] developed deep vein thrombosis followed by pulmonary embolism that required protracted hospital recovery and low molecular weight heparin (LMWH) therapy. Another patient, a 19-year-old male, developed septic arthritis that did not require arthroscopic washout and that resolved through prolonged antibiotic therapy and immobilization. However, at follow-up no patients showed graft failure. No peroneal nerve pathologies were observed before or after surgery.

All patients considered the knee improved $(n=4)$ or greatly improved $(n=6)$ and would repeat the procedure under the same circumstance, including those who had postoperative complications. The main VAS score for pain rating was 0.85 (range $0-3$; Table 2 ).

\section{Discussion}

Our purpose in this study is to evaluate the clinical outcome of isolated PLC reconstruction following the fibularbased technique performed in patients with chronic isolated PLC injury. The present study shows good results in terms of Lysholm and IKDC scores for both surgical techniques, nearly normal postoperative knee ROM, and good lateral and posterolateral knee stability observed on Dial test and varus stress test. No correlation was found comparing clinical results by Dial test and varus stress test with Lysholm score and IKDC score.

Furthermore, the main finding in the study was that three patients $(30 \%)$ presented a reduction of $10^{\circ}$ external rotation compared with the contralateral knee; this was present in two patients treated following the double femoral tunnel technique and in one patient treated following the single femoral tunnel technique. Based on Lysholm knee score, these three patients presented good function of the knee and were classified as nearly normal by IKDC score. Reduction of external rotation was already described in literature and was present when the popliteus muscle-tendon unit was reconstructed by a static stabilizing procedure [12]. In a biomechanical study, Markolf et al. [13] observed that, using two different surgical PLC reconstructions (FCL + PT versus FCL + PFL), the amount of external rotation was equivalent in both techniques. Furthermore, for both techniques, the amount of external rotation was significantly less when compared with the intact knee (the lateral joint was slightly overconstrained). In this study the authors also described that, when tensioning the grafts at $30^{\circ}$ knee flexion, decrease of final tibial external rotation was observed when passing from 10 to $30 \mathrm{~N}$ graft tensioning. In all our patients the surgical procedure performed permitted reconstruction of just the static limb of the popliteus complex, suggesting that the limitation of tibial external rotation is not directly correlated with the surgical procedure. Based on the evidence of our study, we could speculate that the reduction of the tibial external rotation observed in three of our patients seems to be due to excessive tensioning of the graft during surgical reconstruction. Even though good subjective and objective results of this small group were observed, we believe that longer follow-up is required to evaluate any 
degenerative joint disease in the group with diminished tibial external rotation. Moreover, further studies are needed to evaluate appropriate graft tensioning in order to reduce the risk of overconstraining the lateral knee joint.

Isolated PLC injuries are rare, as $90 \%$ of all PLC tears occur in settings with other concomitant ligament injury [14]. Recently, in an MRI study of incidence of ligament injuries [15], it was observed that PLC tears occurred in $16 \%$ of all ligament injuries, whereas isolated PLC tears occurred in $13 \%$ of overall posterolateral knee injuries.

Kannus [16] reported good and excellent results following conservative treatment of grade II PLC injuries, even if residual lateral laxity was common. Worse clinical results were observed for untreated grade III PLC injuries, and posttraumatic arthritis was found in $50 \%$ of patients in an X-ray follow-up study. However, it is a common opinion that ligament tears and secondary joint laxity lead to significant predisposition to articular cartilage degeneration secondary to altered knee forces. Furthermore, it was observed that high adduction moment on PLC-deficient knees could significantly increase the risk of ACL or PCL graft failure [17]. This occurrence could also be assumed for the native ACL and PCL.

Many authors recommend acute primary repair through direct suture of the damaged structures of the PLC to be performed within 2 weeks of injury. Indeed, it has been found that, after 3 weeks, there is significant development of scar tissue planes along the posterolateral knee [18]. However, it was observed that popliteal tears are frequently located at the muscolotendinous junction, precluding direct repair in acute cases [19].

Several surgical procedures have been described in literature, such as advancement techniques (advancement of PT and FCL insertion), augmentation techniques (using the iliotibial band and/or a central slip of biceps tendon), and biceps tenodesis techniques [11, 20]. Recently, following increased anatomical and biomechanical knowledge of the PLC's key structures, various free graft surgical reconstruction techniques have been described [3, $9,10,21]$ that attempt functional restoration of the three key structures of the posterolateral corner (FCL, PFL, and PT). Because of the different surgical procedures described, there is no consensus regarding the ideal reconstruction. Furthermore, PLC surgery is generally performed in a setting with ACL or PCL reconstruction, and very little literature is available describing clinical results of isolated PLC reconstruction. Stannard et al. [22] reported the clinical results of seven isolated PLC reconstructions following the two-tailed modified procedure. In that study, the authors observed that at follow-up the average score for the varus stress test and Dial test was, respectively, 0.1 and 0.3 . The authors also observed no graft failures and that clinical findings on the IKDC form were normal $(n=4)$ or nearly normal $(n=3)$, with good posterolateral knee stability at follow-up. The only two graft failures were observed in patients who underwent combined ACL-PLC reconstruction following knee dislocation. These results are comparable to ours; in fact, in our case series, no graft failure was observed, and on the IKDC form three knees were classified as normal while seven were classified as nearly normal.

In 2003, Kim et al. [23] reported good results with an average postoperative Lysholm knee score of 93.6 (range 65-100) in a case series of 21 isolated PLC reconstructive surgeries using biceps tenodesis that included also ACL and PLC stretching injuries. Yoon et al. [24] reviewed 46 patients having PLC reconstruction, comparing the clinical results of PLC sling procedure with an anatomical PLC reconstruction. In that case series there were four isolated PLC injuries, but the authors did not describe the surgical procedure and clinical results for each case.

Recently, Arthur et al. [25] reported that open wedge osteotomy should be the first stage of treatment in patients with chronic isolated PLC injuries if genu varus alignment is identified. The authors observed that four of six patients with isolated PLC injuries who underwent a first-stage corrective proximal tibial open wedge osteotomy did not require a second-stage ligament reconstruction. Almost all of our patients presented nearly normal knee alignment, while one patient $(n=3)$ presented moderate varus alignment. However, at the time of follow-up assessment, $\mathrm{X}$-ray knee examination for evaluation of the mechanical axis was not performed because the patient did not provide informed consent.

The major limitations of our study are its retrospective nature and its small size. This could be justified by the rarity of the injury. However, our population group was similar in terms of size to other studies reporting results of isolated PLC reconstruction [22-24]. Furthermore, we believe that this small group study provides statistical results that could have been different if the study were conducted on a wider scale. Other limitations include heterogeneity due to the use of two different surgical techniques for femoral graft fixation, PCL status, use of both allograft and autograft, and lack of X-ray checkups. Furthermore, the short follow-up period does not allow us to evaluate differences between these variable in terms of knee function and return to sports.

In conclusion, fibular-based PLC reconstruction showed acceptable short-term results in a small series of patients with isolated PLC injury. Longer follow-up is necessary to determine long-term graft function as well as incidence of degenerative joint disease. Also, further studies are required to optimize the amount of graft tensioning during PLC reconstruction to reduce incidence of overconstraining of the lateral knee joint. 
Acknowledgment The study was authorized by the local ethical committee and was performed in accordance with the ethical standards of the 1964 Declaration of Helsinki as revised in 2000.

Conflict of interest All authors have disclosed all financial support for this work and other potential conflict of interests related to the publication of this manuscript.

\section{References}

1. Andrews JR, Baker C, Curl W, Gidumal R (1988) Surgical repair of acute and chronic lesions of the lateral capsular ligamentous complex of the knee. In: Feagin JA Jr (ed) The crucial ligaments. Churchill Livingston, pp 425-438

2. Gollehon DL, Torzilli PA, Warren RF (1987) The role of the posterolateral and cruciate ligaments in the stability of the human knee. A biomechanical study. J Bone Joint Surg Am 69:233-242

3. LaPrade RF, Ly TV, Wentorf FA, Engebretsen L (2003) The posterolateral attachments of the knee: a qualitative and quantitative morphologic analysis of the fibular collateral ligament, popliteus tendon, popliteofibular ligament, and lateral gastrocnemius tendon. Am J Sports Med 31:854-860

4. Frank JB, Youm T, Meislin RJ, Rokito AS (2007) Posterolateral corner injuries of the knee. Bull NYU Hosp Joint Dis 65:106-114

5. Maynard MJ, Deng X, Wickiewicz TL, Warren RF (1996) The popliteofibular ligament. Rediscovery of a key element in posterolateral stability. Am J Sports Med 24:311-316

6. Fanelli GC, Larson RV (2002) Practical management of posterolateral instability of the knee. Arthroscopy 18:1-8

7. Hughston JC, Andrews JR, Cross MJ, Moschi A (1976) Classification of knee ligament instabilities. Part II. The lateral compartment. J Bone Joint Surg Am 58:173-179

8. LaPrade RF (1997) Arthroscopic evaluation of the lateral compartment of knees with grade 3 posterolateral knee complex injuries. Am J Sports Med 25:596-602

9. McGuire DA, Wolchok JC (2003) Posterolateral corner reconstruction. Arthroscopy 19:790-793

10. Arciero RA (2005) Anatomic posterolateral corner knee recostruction. Arthroscopy 21:1147

11. Hughston JC, Jacobson KE (1985) Chronic posterolateral rotatory instability of the knee. J Bone Joint Surg Am 67:351-359

12. Yoon KH, Bae DK, Ha JH, Park SW (2006) Anatomic reconstructive surgery for posterolateral instability of the knee. Arthroscopy 22:159-165
13. Markolf KL, Graves BR, Sigward SM, Jackson SR, McAllister DR (2007) Effects of posterolateral reconstructions on external tibial rotation and forces in a posterior cruciate ligament graft. J Bone Joint Surg Am 89:2351-2358

14. DeLee JC, Riley MB, Rockwood CA Jr (1983) Acute posterolateral rotatory instability of the knee. Am J Sports Med 11:199207

15. LaPrade RF, Wentorf FA, Fritts H, Gundry C, Hightower CD (2007) A prospective magnetic resonance imaging study of the incidence of posterolateral and multiple ligament injuries in acute knee injuries presenting with a hemarthrosis. Arthroscopy 23:1341-1347

16. Kannus P (1989) Non operative treatment of grade II and III sprains of the lateral ligament compartmen of the knee. Am J Sport Med 17:83-88

17. Harner CD, Vogrin TM, Höher J, Ma CB, Woo SL (2000) Biomechanical analysis of a posterior cruciate ligament reconstruction. Deficiency of the posterolateral structures as a cause of graft failure. Am J Sport Med 28:32-39

18. LaPrade RF, Wentorf F (2002) Diagnosis and treatment of posterolateral knee injuries. Clin Orthop Relat Res 402:110-121

19. Clancy WG Jr, Shepard MF, Cain EL Jr (2003) Posterior lateral corner reconstruction. Am J Orthop 32:171-176

20. Clancy WG (1988) Repair and reconstruction of the posterior cruciate ligament. In: Chapman M (ed) Operative orthopaedics. JB Lippincott, Philadelphia, pp 1651-1665

21. Larsen MW, Moinfar AR, Moorman CT 3rd (2005) Posterolateral corner reconstruction: fibular-based technique. J Knee Surg 18:163-166

22. Stannard JP, Brown SL, Robinson JT, McGwin G Jr, Volgas DA (2005) Reconstruction of the posterolateral corner of the knee. Arthroscopy 21:1051-1059

23. Kim SJ, Shin SJ, Jeong JH (2003) Posterolateral rotatory instability treated by a modified biceps rerouting technique: technical considerations and results in cases with and without posterior cruciate ligament insufficiency. Arthroscopy 19:493-499

24. Yoon KH, Bae DK, Ha JH, Park SW (2006) Anatomic reconstructive surgery for posterolateral instability of the knee. Arthroscopy 22:159-165

25. Arthur A, LaPrade RF, Agel J (2007) Proximal tibial opening wedge osteotomy as the initial treatment for chronic posterolateral corner deficiency in the varus knee: a prospective clinical study. Am J Sports Med 35:1844-1850 